\title{
Protective effects of olive leaf extract on inflammatory activation of endothelial cells
}

B. Burja ${ }^{1,2}$, T. Kuret ${ }^{1,3}$, D. Topalovič ${ }^{4}$, L. Živkovič4 ${ }^{4}$ K. Mrak-Poljšak ${ }^{1}$, T. Janko ${ }^{5}$, B. Spremo-Potparevič ${ }^{4}$, P. Žigon ${ }^{1}$, S. Čučnik ${ }^{1,3}$, S. Sodin-Semrl'1,5, K. Lakota1,5, M. Frank-Bertoncelj²

${ }^{1}$ Department of Rheumatology, University Medical Centre Ljubljana, Slovenia; ${ }^{2}$ Center of Experimental Rheumatology, University Hospital Zurich, Switzerland ${ }^{3}$ Department of Pathobiology, Faculty of Pharmacy, University of Belgrade, Serbia; ${ }^{4}$ Chair of Clinical Biochemistry, Faculty of Pharmacy, University of Ljubljana, Slovenia ${ }^{5}$ Faculty of Mathematics, Natural Sciences and Information Technologies, University of Primorska, Slovenia

\section{BACKGROUND}

Olive leaf extract (OLE) has been traditionally used due to its anti-inflammatory and anti-atherogenic effects. The mechanism through which OLE modulates atherogenesis is unclear. Serum amyloid A (SAA) is a major inflammatory mediator in humans linking inflammation and atherosclerosis.

\section{OBJECTIVE}

To explore the anti-inflammatory properties of OLE in the SAA-activated endothelia cells from the human coronary arteries (HCAEC), the most susceptible vascular site in atherosclerosis.

\section{METHODS}

HCAEC were treated with SAA $(1000 \mathrm{nM} ; 24 \mathrm{~h})$ and/or OLE $(0.5$ and $1.0 \mathrm{mg} / \mathrm{ml} ; 45$ min prior SAA). The amount of secreted IL-6 and IL-8 was quantified by ELISA. The expression of VCAM-1, E-selectin, matrix metalloproteinases (MMP2 and MMP9) and miRNAs 146a and let-7e was determined by qPCR. Phosphorylation of NF-KB was assessed by Western blot and DNA damage was measured using the COMET assay.

\section{OLE regulates SAA-induced miR-146a and let-7e}
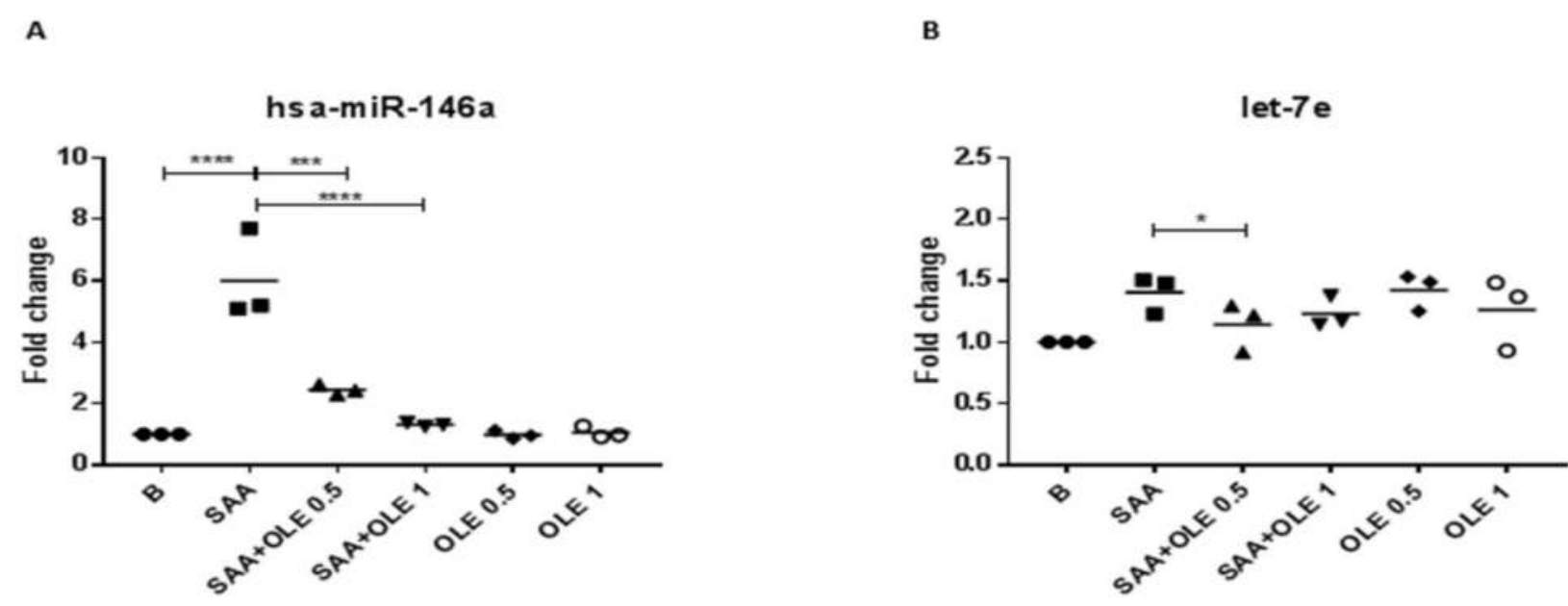

The mean fold changes of mRNA expression levels of miR-146a (A) and let-7e (B) are shown for 3 biological replicates.

\section{OLE attenuates SAA-induced and $\mathrm{H} 2 \mathrm{O} 2$-induced DNA damage in HCAEC}
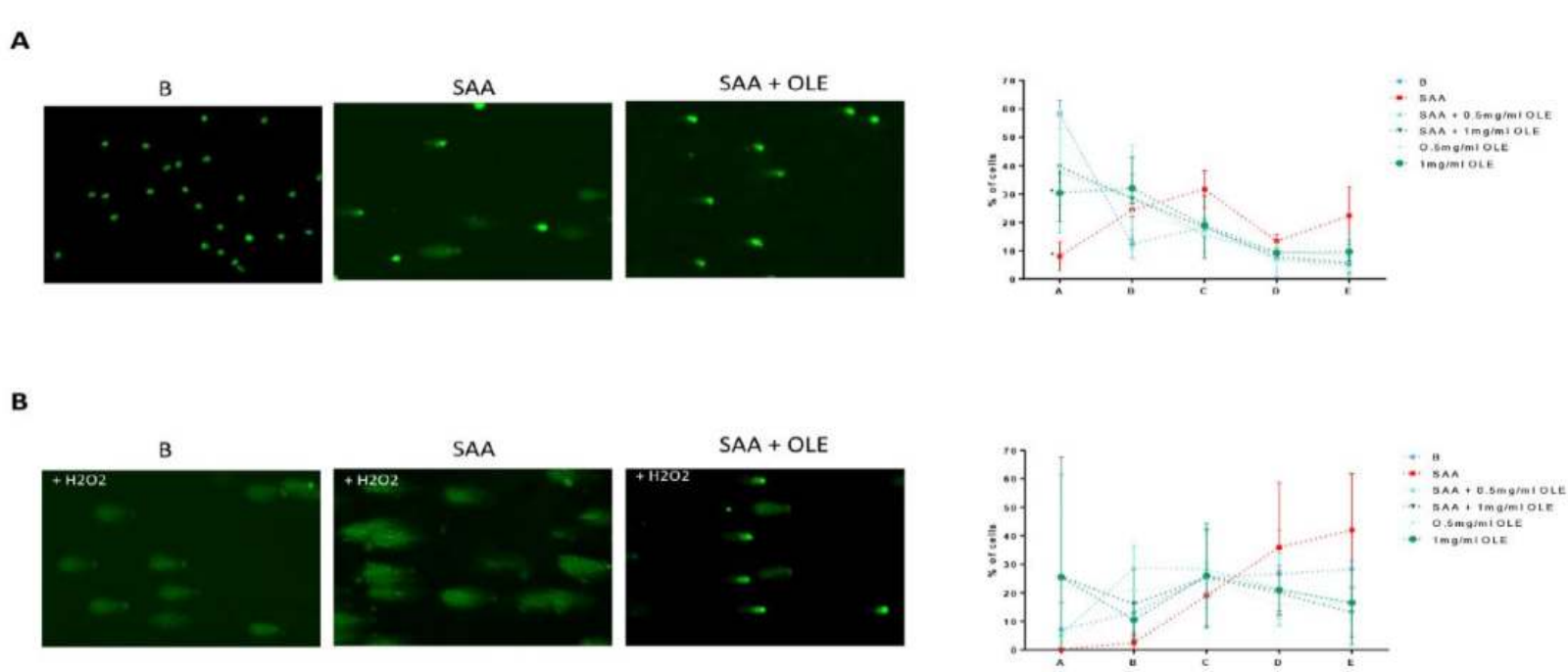

COMET assay was performed without (A) and with oxidative treatment $\left(3 \% \mathrm{H}_{2} \mathrm{O}_{2}\right)(\mathrm{B})$. DNA damage wa evaluated according to the following categories: A - no DNA damage, B - slight tail, $\mathrm{C}$ - strong signal in tail and nucleus, D - majority of DNA in tail, E - no DNA in round nucleus.
OLE decreases SAA-driven release of pro-inflammatory cytokine IL-6 and chemokine IL-8 from HCAEC
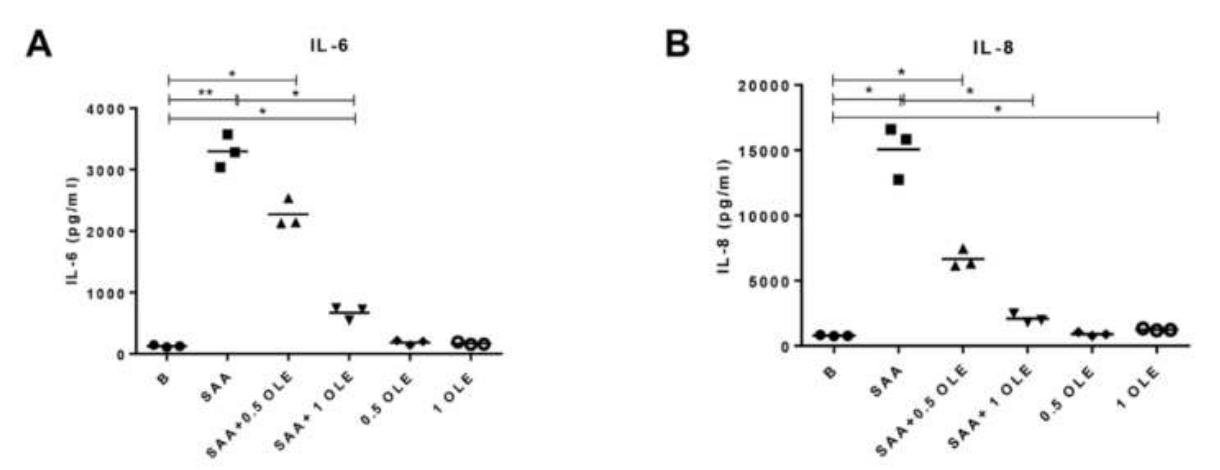

The amount of IL-6 (A) and IL-8 (B) in cell culture supernatants was measured by ELISA. The mean of 3 biological replicates is shown.

OLE decreases mRNA expression of adhesion molecules VCAM-1 and E selectin and MMP2 in HCAEC
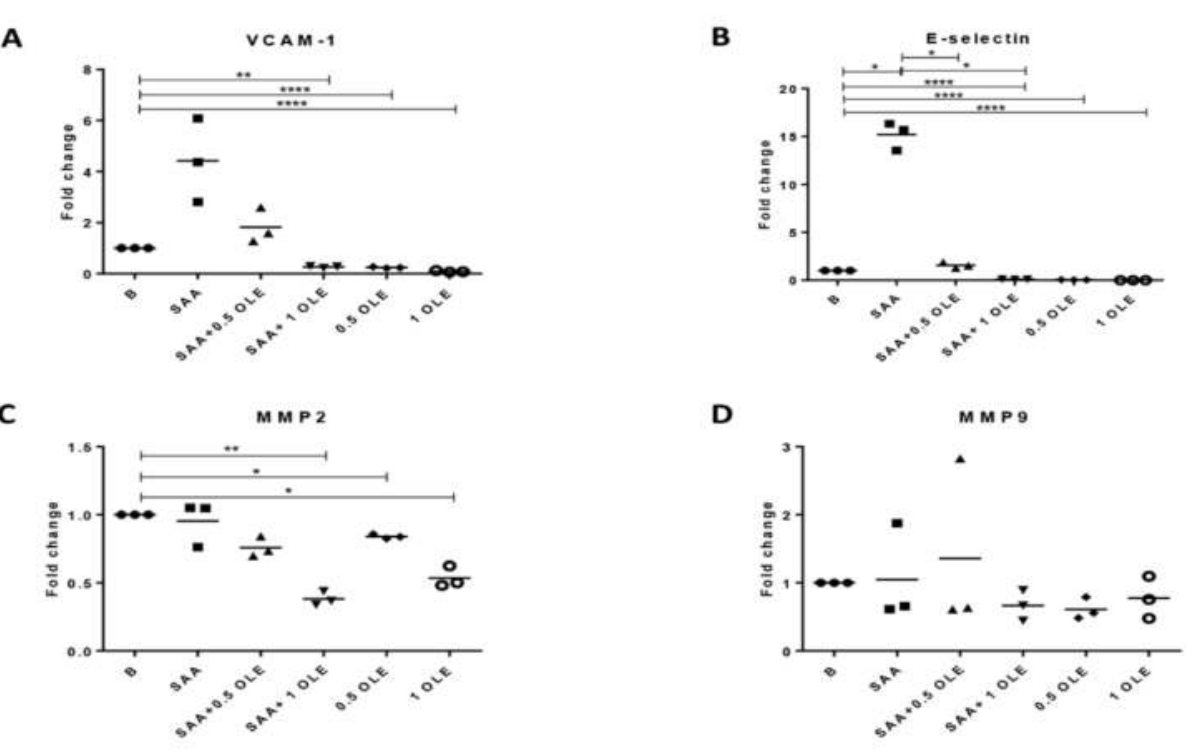

The expression of VCAM-1 (A), E-selectin (B), MMP2 (C) and MMP9 (D) mRNA was determined by qPCR. Mean of 3 biological replicates is shown.

\section{RESULTS}

OLE decreased significantly released protein levels of IL-6 and IL-8, as well as mRNA expression of E-selectin in SAA-stimulated HCAEC and reduced MMP2 levels in unstimulated cells. Phosphorylation of NF- $\kappa \mathrm{B}$ was upregulated in the presence of SAA, with OLE significantly attenuating this effect. OLE stabilized SAA-induced upregulation of microRNA-146a and let-7e and protected HCAEC from SAA- and $\mathrm{H}_{2} \mathrm{O}_{2}$-driven DNA damage.

OLE attenuates SAA-induced phosphorylation of NF-KB.

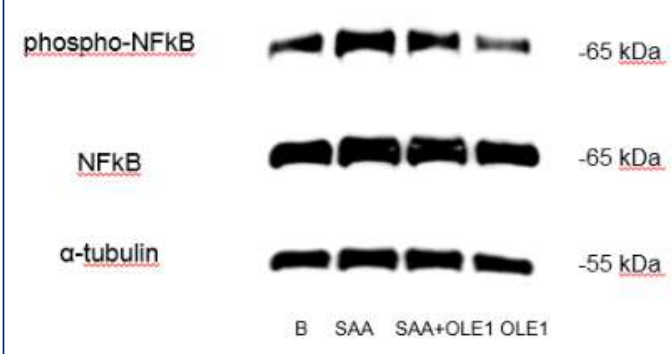

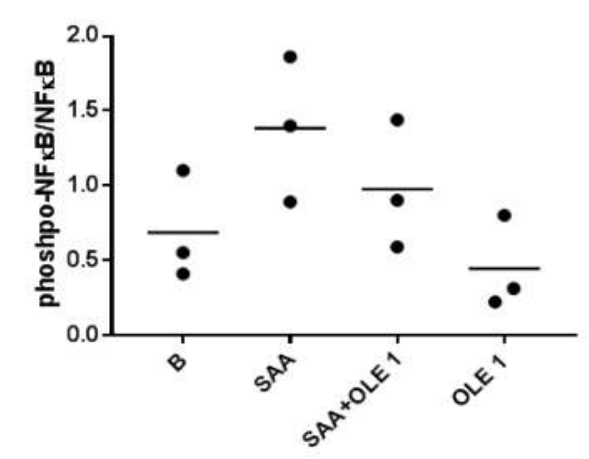

Western blot shows phosphorylation of NF-KB in HCAEC pretreated with OLE for $1 \mathrm{~h}$ and then treated with/without SAA for 1 h.

\section{CONCLUSION}

OLE significantly attenuated certain pro-inflammatory and pro-adhesive responses and decreased DNA damage in HCAEC upon stimulation with SAA. The reversal of SAA-driven endothelial activation by OLE might contribute to its anti-inflammatory and anti-atherogenic effects in HCAEC. 